\title{
Efeitos da inclusão de torta de macaúba sobre a população de protozoários ruminais de caprinos
}

\author{
Luana Marta de Almeida Rufino ${ }^{1}$, Silene Maria Prates Barreto ${ }^{1}$, Eduardo Robson Duarte ${ }^{1}$, \\ Luciana Castro Geraseev ${ }^{1}$, Antônio Carlos Ramos Santos ${ }^{1}$, Yuri Genaro Jaruche ${ }^{1}$ \\ 1 Instituto de Ciências Agrárias, Universidade Federal de Minas Gerais. Av. Universitária, 1000, Bairro Universitário, Montes Claros, \\ 39400-006, MG, Brasil.
}

RESUMO - Nesta pesquisa avaliaram-se os efeitos de diferentes níveis de inclusão da torta de macaúba (Acrocomia aculeata) sobre a população de protozoários do rúmen de caprinos. Foram utilizados oito cabritos machos castrados, sem raça definida, portadores de fístulas ruminais, dispostos em dois quadrados latinos e alimentados com quatro dietas com diferentes níveis da torta. Para quantificação dos protozoários, amostras de $1 \mathrm{~mL}$ do líquido ruminal de cada animal foram diluídas em $9 \mathrm{~mL}$ de solução de formaldeído a $10 \%$. Após diluições decimais sucessivas em solução salina, os protozoários foram quantificados em câmara de Sedgewick. A inclusão de torta de macaúba no nível de 5\% da dieta não alterou a concentração média dos protozoários no líquido ruminal em comparação ao grupo controle ( $0 \%$ de inclusão). As dietas com 10 e $15 \%$ de torta promoveram aumento significativo da concentração média de protozoários e não reduziram a concentração de protozoários dos outros grupos. O perfil das populações de protozoários foi alterado somente em três dos dezessete gêneros detectados, comprovando diversidade no ecossistema ruminal e sugerindo que esse subproduto pode ser uma alternativa segura para esses microrganismos ruminais.

Palavras-chave: Acrocomia aculeata, pequenos ruminantes, rúmen, semiárido, subproduto

\section{Effects of inclusion of macaúba cake on ruminal protozoa population of goats}

\begin{abstract}
The objective of this work was to evaluate the effects of different levels of inclusion of macauba (Acrocomia aculeata) cake on the protozoa population in the rumen of goats. It was used eight castrated undefinied breed goats with rumen cannula arranged in two Latin squares and fed four diets with different levels of macauba cake. For quantification of protozoa, 1-mL samples of the rumen fluid of each animal were diluted in $9 \mathrm{ml}$ of formaldehyde solution at $10 \%$. After successive decimal dilutions in saline solution, protozoa were quantified in Sedgewick chambers. The inclusion of macauba cake at the level of $5 \%$ of the diet did not change the average protozoa concentration in the rumen fluid, compared with the control group $(0 \%$ of inclusion). Diets with 10 and 15\% of macauba cake promoted significant increase of average concentration of protoza and did not reduce the concentrations of protozoa from other groups. The profile of protozoa populations was changed only in three out of 17 detected genera, proving that there is diversity in the ruminal ecosystem and it suggests that this by-product can be a safe alternative for those rumen microorganisms.
\end{abstract}

Key Words: Acrocomia aculeata, byproduct, rumen, semi-arid, small ruminants

\section{Introdução}

A pecuária constitui a base da economia do Norte de Minas, porém é necessária a implantação de técnicas produtivas que permitam a convivência com os efeitos da estiagem prolongada. Em regiões com essas condições climáticas, estudos para a utilização de alimentos regionais alternativos como forma de suplementação ao rebanho são imprescindíveis para se alcançar melhores índices de produtividade e aumento da renda familiar dos produtores desse setor (Geraseev et al., 2007).
Os resíduos agroindustriais representam recurso viável para alimentação dos ruminantes. A torta da macaúba (Acrocomia aculeata (Jacq.) Lodd. Ex Mart.), um resíduo da extração do óleo da polpa do coco, pode ser uma alternativa promissora para uso na alimentação de ruminantes principalmente em regiões semiáridas. Análises preliminares comprovam que a torta de macaúba possui teor de fibra em detergente neutro próximo de 60,0\% e extrato etéreo próximo a $10 \%$, o que poderia ocasionar limitação na ingestão de matéria seca e alterações na fermentação ruminal (Barreto, 2008). 
O rúmen contém diferentes reinos e classes de microrganismos, como protozoários, fungos, bactérias e vírus, os quais estabelecem diversas interações em um complexo ecossistema (Dehority, 1984; Dehority \& Tirabasso 1989; Kamra, 2005). Os protozoários foram os primeiros microrganismos a serem descritos neste nicho. Podem representar 2\% de peso do conteúdo ruminal, $40 \%$ do nitrogênio total e $60 \%$ do produto final da fermentação (Kamra, 2005).

Em dietas ricas em óleo, a população dos protozoários decresce significativamente, reduzindo também a atividade celulolítica (Valinote et al., 2005, Kamra, 2005). Segundo Lopes et al. (2002), protozoários ruminais possuem considerável atividade celulolítica e fermentativa e animais faunados apresentam maior ganho de peso e digestibilidade quando comparados com os defaunados.

O excesso de óleo presente na torta de macaúba poderia comprometer a fermentação ruminal e o desempenho animal. Por esse motivo, avaliações dos efeitos da inclusão desse alimento sobre a microbiota ruminal, em especial sobre os protozoários ciliados, são fundamentais para se fazerem recomendações de uso desse subproduto em dieta para ruminantes.

Objetivou-se com este estudo avaliar os efeitos da inclusão de torta de macaúba sobre a população de protozoários ruminais em caprinos da região semiárida do norte de Minas Gerais.

\section{Material e Métodos}

O experimento foi conduzido nas dependências do setor de Caprinocultura do Departamento de Zootecnia do Instituto de Ciências Agrárias da Universidade Federal de Minas Gerais, em Montes Claros, no período de maio a agosto de 2007. Foram utilizados oito caprinos adultos, machos castrados, sem padrão racial definido, portando cânulas ruminais permanentes. O delineamento experimental utilizado foi de dois quadrados latinos $4 \times 4$. O experimento teve duração de 84 dias, divididos em quatro períodos de 21 dias, com 14 dias para adaptação da microbiota ruminal à dieta e 7 dias para a coleta de dados.

As dietas foram elaboradas na forma de dieta total (silagem + concentrado), balanceadas de acordo com as recomendações do NRC (1981), para ganho estimado de 100 g/dia (Tabela 1). Antes do período experimental, os animais foram identificados, vermifugados e vacinados contra clostridioses e, durante o experimento, foram mantidos alojados em baias individuais providas de comedouros e de bebedouros.

Amostras de $20 \mathrm{~mL}$ do suco ruminal foram coletadas, homogeneizadas e alíquotas de 1,0 mL foram transferidas para tubos contendo $9,0 \mathrm{~mL}$ de formaldeído a $10 \%$, para a conservação dos protozoários. Posteriormente, foram realizadas diluições sucessivas em solução salina, para determinação do número de protozoários em câmaras de Sedgewick. As concentrações de pequenos, médios e grandes protozoários por mililitro de suco ruminal foram determinadas após a visualização em microscópio óptico, utilizando-se a objetiva de 10X (Dirksen, 1993; Franzolin et al., 2000). A identificação desses protozoários foi realizada em lâminas e lamínulas com adição de uma gota da amostra em solução de $10^{-1}$ e uma gota de solução de lugol. A leitura e identificação foram realizadas sob a luz da microscopia óptica, utilizando a objetiva de 40X para caracterizar o mínimo de 200 indivíduos por amostra. Os gêneros foram classificados de acordo com as características morfológicas descritas por Dehority (1984), adaptado por D’Agosto \& Carneiro (1999).

Após a transformação logarítmica (log x em base 10), os dados foram submetidos à análise de variância,

Tabela 1 - Composição das dietas experimentais

\begin{tabular}{|c|c|c|c|c|}
\hline \multirow[t]{2}{*}{ Ingrediente } & \multicolumn{4}{|c|}{ Nível de torta de macaúba na dieta } \\
\hline & $0 \%$ & $5 \%$ & $10 \%$ & $15 \%$ \\
\hline Silagem (\%) & 65,17 & 60,15 & 56,00 & 51,45 \\
\hline Milho (\%) & 11,10 & 14,20 & 16,12 & 18,78 \\
\hline Caroço de algodão (\%) & 14,78 & 9,80 & 4,97 & 0,00 \\
\hline Farelo de soja (\%) & 8,10 & 10,00 & 11,96 & 13,83 \\
\hline Torta de macaúba (\%) & 0,00 & 5,00 & 10,00 & 15,15 \\
\hline Premix (\%) & 0,850 & 0,848 & 0,808 & 0,785 \\
\hline \multicolumn{5}{|l|}{ Composição nutricional calculada } \\
\hline Matéria seca (\%) & 54,78 & 57,64 & 59,62 & 62,07 \\
\hline Proteína bruta (\%) & 12,72 & 12,67 & 12,65 & 12,57 \\
\hline Nutrientes digestíveis totais $(\%)^{1}$ & 63,05 & 63,31 & 63,32 & 63,44 \\
\hline Extrato etéreo(\%) & 5,25 & 4,69 & 4,15 & 3,59 \\
\hline Fibra em detergente neutro (\%) & 47,74 & 46,30 & 45,45 & 44,28 \\
\hline
\end{tabular}


utilizando-se o Sistema de Análises Estatísticas e Genéticas SAEG (2007). As médias das concentrações de protozoários pequenos, médios, grandes e do número total, em função dos quatro tratamentos utilizados, foram comparadas pelo teste de Scott Knott, com nível de 5\% de significância. Para a análise do perfil da população, as porcentagens dos gêneros identificados sofreram transformação radical (raiz de $\mathrm{x}+1)$.

Os procedimentos adotados com os animais neste trabalho estiveram de acordo com os princípios éticos da experimentação animal, aprovados no protocolo 160/2006 pelo Comitê de Ética em Experimentação animal da UFMG.

\section{Resultados e Discussão}

A torta de macaúba (Acrocomaia aculeata) é um resíduo que apresenta baixo teor de proteína e alto teor de fibra e energia e que, teoricamente, poderia ser aproveitada na alimentação de ruminantes, especialmente para animais em crescimento, dado o alto teor de extrato etéreo na matéria seca (18,88\%) (Barreto, 2008). Em análise preliminar, não houve alterações significativas decorrentes da inclusão desse subproduto nas características macroscópicas e físicas, no potencial de redução do azul de metileno, no pH e na concentração do nitrogênio amoniacal do líquido ruminal de caprinos (Barreto, 2008).

Neste estudo, a população de protozoários médios aumentou significativamente $(\mathrm{P}<0,05)$ a partir do nível $10 \%$ de inclusão da torta de macaúba (Tabela 2). Quando fornecidas dietas ricas em extrato etéreo, ocorre significativa redução na população de protozoários no ecossistema ruminal (Valinote et al., 2005). Apesar de ser rica em óleo, à medida que a torta da macaúba foi incluída na dieta, ocorreu diminuição do teor de extrato etéreo total da dieta, o que pode ter ocasionado esse aumento na população dos médios protozoários.

Martinele et al. (2008) observaram significativa redução das populações do gênero Entodinium no ambiente ruminal quando fornecidas dietas contendo óleo de soja, associado ou não a monensina, o que indica efeito tóxico dos lipídios do óleo de soja sobre os protozoários desse gênero. Além da redução nos organismos do gênero Entodinium, o óleo de soja também reduziu significativamente a contagem dos gêneros Dasytricha, Eremoplastron e Isotricha.

As concentrações médias de protozoários neste experimento, que corresponderam a $9,5 \times 10^{5} / \mathrm{mL}$, foram próximas àquelas encontradas por Franzolin \& Franzolin (2000) em estudo no qual avaliaram o líquido ruminal de búfalos e bovinos alimentados com uma dieta à base de cana-de-açúcar. Esses autores encontraram valores médios de $4,85 \times 10^{5} / \mathrm{mL}$ para bovinos e $3,82 \times 10^{5} / \mathrm{mL}$ para búfalos .

Após identificação e análise da distribuição dos 17 gêneros observados nas amostras avaliadas (Tabela 3), observou-se que, nos animais mantidos com a dieta sem torta de macaúba e com aquela contendo 30\% desse subproduto, houve predominância do gênero Entodinium, que correspondeu a 31,02\% e 28,8\% dos protozoários identificados, respectivamente. Por outro lado, com a inclusão de $5 \%$ de torta na dieta, os gêneros mais frequentes foram Entodinium (22,09\%) e Eremoplastron (23,8\%), e com $10 \%$ de inclusão, ocorreu predominância do gênero Charonina (28,6\%).

O gênero Entodinium pode representar até $90 \%$ da população de ciliados no rúmen de ovinos alimentados com cana-de-açúcar, segundo Franzolin \& Franzolin (2000). Outros estudos indicam também grande variação na população de protozoários do rúmen em diferentes espécies de ruminantes e mesmo entre animais de mesma espécie (Bird et al., 1979; Franzolin, 1988; Rispoli et al., 2009). Coalho et al. (2003) estudaram protozoários ciliados em bovinos que consumiam dietas com diferentes níveis de proteína não-degradável no rúmen e constataram que a inclusão de ureia aumentou a população de ciliados, com predominância do gênero Entodinium.

Estudos têm comprovado a presença de atividades hemicelulolítica e celulolítica nos ciliados do rúmen, especialmente do grupo dos grandes entodiniomorfos (Frazolin \& Franzolin, 2000). Segundo Orpin \& Letcher(1978), os grandes protozoários ciliados colonizam fragmentos da fibra, ingerem diretamente os tecidos da planta e facilitam a ação de bactérias específicas.

Tabela 2 - Contagem média de protozoários ruminais (x 105\% mL) em caprinos alimentados com dietas contendo torta de macaúba

\begin{tabular}{|c|c|c|c|c|c|}
\hline \multirow[b]{2}{*}{ Protozoários } & \multicolumn{4}{|c|}{ Nível de torta de macaúba na dieta } & \multirow[t]{2}{*}{ CV (\%) } \\
\hline & $0 \%$ & $5 \%$ & $10 \%$ & $15 \%$ & \\
\hline Pequenos & $5,4 a$ & $5,96 a$ & $5,92 \mathrm{a}$ & 6,53a & 32,94 \\
\hline Médios & $1,96 b$ & $2,13 b$ & $2,78 \mathrm{a}$ & $2,81 \mathrm{a}$ & 44,08 \\
\hline Grandes & $0,92 \mathrm{a}$ & $1,02 \mathrm{a}$ & $1,29 a$ & 1,39a & 38,82 \\
\hline Total & $8,28 a$ & $9,10 \mathrm{a}$ & 9,99a & $10,72 \mathrm{a}$ & 20,98 \\
\hline
\end{tabular}

Os dados foram transformados (Log x ) para as análises estatísticas. Médias seguidas de mesma letra minúscula na linha não diferem entre si a 5\% pelo teste Scott-Knott. 
Tabela 3 - Distribuição percentual de protozoários ruminais em caprinos alimentados com dietas contendo torta de macaúba

\begin{tabular}{lcccc}
\hline & \multicolumn{4}{c}{ Nível de torta de macaúba na dieta } \\
\cline { 2 - 5 } Gênero & $0 \%$ & $5 \%$ & $10 \%$ & $15 \%$ \\
\hline Buetschilia spp. & $0,77 \mathrm{a}$ & $0,37 \mathrm{a}$ & $0,12 \mathrm{a}$ & $0,06 \mathrm{a}$ \\
Isotricha spp. & $0,55 \mathrm{a}$ & $0,40 \mathrm{a}$ & $0,74 \mathrm{a}$ & $0,79 \mathrm{a}$ \\
Dasytricha spp. & $5,17 \mathrm{a}$ & $4,10 \mathrm{a}$ & $4,40 \mathrm{a}$ & $4,80 \mathrm{a}$ \\
Charonina spp. & $14,12 \mathrm{~b}$ & $14,79 \mathrm{~b}$ & $28,61 \mathrm{a}$ & $11,72 \mathrm{~b}$ \\
Entodinium spp. & $31,02 \mathrm{a}$ & $22,09 \mathrm{~b}$ & $18,74 \mathrm{~b}$ & $28,80 \mathrm{a}$ \\
Diplodinium spp. & $4,29 \mathrm{a}$ & $4,08 \mathrm{a}$ & $3,76 \mathrm{a}$ & $4,20 \mathrm{a}$ \\
Eodinium spp. & $15,07 \mathrm{a}$ & $16,06 \mathrm{a}$ & $12,96 \mathrm{a}$ & $18,54 \mathrm{a}$ \\
Eremoplastron spp. & $16,01 \mathrm{a}$ & $23,79 \mathrm{a}$ & $14,60 \mathrm{a}$ & $17,27 \mathrm{a}$ \\
Eudiplodinium spp. & $0,98 \mathrm{a}$ & $0,63 \mathrm{a}$ & $0,00 \mathrm{a}$ & $2,23 \mathrm{a}$ \\
Diploplastron spp. & $2,46 \mathrm{a}$ & $3,98 \mathrm{a}$ & $2,20 \mathrm{a}$ & $1,86 \mathrm{a}$ \\
Polyplastron spp. & $3,04 \mathrm{a}$ & $1,80 \mathrm{a}$ & $1,11 \mathrm{a}$ & $1,67 \mathrm{a}$ \\
Ostracodinium spp. & $2,29 \mathrm{a}$ & $1,67 \mathrm{a}$ & $1,92 \mathrm{a}$ & $2,19 \mathrm{a}$ \\
Elytroplastron spp. & $0,31 \mathrm{a}$ & $0,00 \mathrm{a}$ & $0,00 \mathrm{a}$ & $0,08 \mathrm{a}$ \\
Metadinium spp. & $0,45 \mathrm{a}$ & $0,00 \mathrm{a}$ & $1,25 \mathrm{a}$ & $0,54 \mathrm{a}$ \\
Enoploplastron spp. & $0,31 \mathrm{a}$ & $0,92 \mathrm{a}$ & $2,16 \mathrm{a}$ & $0,58 \mathrm{a}$ \\
Ophyriscilex spp. & $0,79 \mathrm{a}$ & $1,31 \mathrm{a}$ & $1,42 \mathrm{a}$ & $1,57 \mathrm{a}$ \\
Epidinium spp. & $2,35 \mathrm{a}$ & $4,01 \mathrm{a}$ & $6,02 \mathrm{a}$ & $3,11 \mathrm{a}$ \\
\hline
\end{tabular}

Para as análises estatísticas, dados de porcentagens obtidos sofreram transformações radicais $(\sqrt{x}+1)$.

As médias seguidas de mesma letra na linha não diferiram entre os níveis de macaúba incluídos na dieta quando comparadas estatisticamente pelo teste de Skott Knott a 5\% de probabilidade.

Neste experimento, além do predomínio dos gêneros citados anteriormente, foi observada diversidade da população de protozoários do rúmen, com um total de 17 gêneros observados para as diferentes dietas utilizadas, comprovando equilíbrio no ecossistema ruminal. Foi constatado também número significativo de grandes protozoários que são os mais sensíveis às mudanças do ecossistema ruminal (Kamra, 2005), e isso sugere que a inclusão de torta de macaúba pode ser uma alternativa segura para a população desses microrganismos.

\section{Conclusões}

A inclusão de torta de macaúba na dieta nos níveis de 10 e 15\% aumenta a população de médios protozoários e não reduz a população dos demais grupos. O perfil das populações de protozoários somente é alterado no caso dos gêneros Entodinium e Charonina em um total de 17 gêneros detectados na microbiota dos animais avaliados.

\section{Agradecimentos}

À Cooperativa de Pequenos Produtores de Riacho D’antas e Adjacências, à Fundação de Amparo a Pesquisa do Estado de Minas Gerais (FAPEMIG), ao Conselho Nacional de Desenvolvimento Científico e Tecnológico (CNPq).

\section{Referências}

BARRETO, S.M.P. Avaliação da torta de macaúba na alimentação de caprinos. 2008. 74f. Dissertação (Mestrado em Agroecologia) - Instituto de Ciências Agrárias da Universidade Federal de Minas Gerais, Montes Claros.

BIRD, S.H.; HILL, M.K.; LENG, R.A. The effects of defaunation of the rumen on the growth of lambs on low-protein-highenergy diets. Bristish Journal Nutrition, v.42, p.81-87, 1979.

COALHO, M.R.; NOGUEIRA FILHO, J.C.M.; CUNHA, J.A. et al. Estudo dos protozoários ciliados em bovinos consumindo dietas com diferentes níveis de proteína não degradável no rúmen. Acta Scientiarum.Animal Sciences, v.25, n.1, p.193-199, 2003.

D’AGOSTO, M.; CARNEIRO, M.E. Evaluation of lugol solution used for counting rumen ciliates. Revista Brasileira de Zoologia, v.16, p.725-729, 1999.

DEHORITY, B.A.; TIRABASSO, P.A. Factors affecting the migration and sequestration of rumen protozoa in family Isotrichiidae. Journal Gen. of Microbiology, v.135, n.3, p.539-548, 1989.

DEHORITY, B.A. Evaluation of subsampling and fixation procedures used for counting rumen protozoa. Applied and Environmental Microbiology, v.48, p.182-185, 1984.

DIRKSEN, G. Sistema digestivo. In: DIRKSEN, G.; GRÜNDER, H.D.; STÖBER, M. (Eds.). Rosenberger: exame clínico dos bovinos. 3.ed. Rio de Janeiro: Guanabara-Koogan, 1993. p.167-169.

FRANZOLIN, M.H.T. Efeitos da cana-de-açúcar (Saccharum officinarum L.) sobre os protozoários ciliados, número de revoluções ruminais e volúme do rúmen, em ovinos. 1988. 48f. Dissertação (Mestrado em Nutrição Animal) - Faculdade de Medicina Veterinária e Zootecnia da Universidade de São Paulo, Pirassununga.

FRANZOLIN, M.H.T.; LUCCI, C.S.; FRANZOLIN, R. Efeitos de rações com níveis crescentes de cana-de-açúcar em substituição à silagem de milho sobre a população de protozoários ciliados no rúmen de ovinos. Revista Brasileira Zootecnia, v.29, n.5, p.1452-1457, 2000.

FRANZOLIN, R.; FRANZOLIN, M.H.T. População protozoários ciliados e degradabilidade ruminal em búfalos e bovinos zebuínos sob dieta à base de cana-de-açúcar. Revista Brasileira Zootecnia, v.29, n.6, p.1853-1861, 2000.

GERASSEEV, L.C.; DUARTE, E.R.; ALMEIDA, A.C. et al. Diagnóstico e recomendações técnicas para ovinocaprinocultura no Norte de Minas. Montes Claros: Instituto de Ciências Agrárias, 2007. 40p.

KAMRA, D.N. Rúmen microbial ecosystem. Current Science, v.89, p.124-134, 2005.

LOPES, F.C.F.; AROEIRA, L.J.M.; ARCURI, P.B. et al. Efeitos da defaunação em ovinos alimentados com cana-de-açúcar (Saccharum officinarum, L.) adicionada de uréia. Arquivo Brasileiro de Medicina Veterinária e Zootecnia, v.54, n.2, p.180-188, 2002.

MARTINELE, I.; EIFERT, E.C.; LANA, R.P. et al. Efeito da monensina e do óleo de soja sobre os protozoários ciliados do rúmen e correlação dos protozoários com parâmetros da fermentação ruminal e digestivos. Revista Brasileira de Zootecnia, v.37, n.6, p.1129-1136, 2008.

NATIONAL RESEARCH COUNCIL - NRC. Nutrients requiremens of goats. Washington, D.C.: National Academy of Science, 1981. 91p.

ORPIN, C.G.; LETCHER, A.J. Some factors controlling the attachment of the rumen holotrich protozoa Isotricha intestinalis and I. prostoma to plant particles in vitro. Journal of General Microbiology, v.106, n.5, p.33-40, 1978.

RISPOLI, T.B.; RODRIGUES, I.L.; MARTINS NETO, R.G. et al. Protozoários ciliados do rúmen de bovinos e bubalinos alimentados com dietas suplementadas com monensina ou própolis. Pesquisa Agropecuária Brasileira, v.44, n.1, p.92-97, 2009. 
UNIVERSIDADE FEDERAL DE VIÇOSA - UFV. Sistema de Análises Estatísticas e Genéticas - SAEG. versão 9.0. Viçosa, MG, 2007. (CD-ROM).

VALINOTE, A.C.; NOGUEIRA FILHO, J.C.M.; LEME, P.R. et al. Fontes de lipídeos e monensina na alimentação de novilhos nelore e sua relação com a população de protozoários ciliados do rúmen. Revista Brasileira Zootecnia, v.34, p.1418-1423, 2005.

WEISS, W.P. Estimating the available energy content of feeds for dairy cattle. In: Symposium: energy availability. Journal of Dairy Science, v.81, p.830-839, 1998. 